\title{
Repeat Stereotactic Body Radiation Therapy (SBRT) for Salvage of Isolated Local Recurrence after Definitive Lung SBRT
}

\author{
William R Kennedy, MD ${ }^{1}$, Prashant Gabani, MD ${ }^{1}$, John Nikitas, BA ${ }^{1}$, Clifford G Robinson, \\ MD ${ }^{1}$, Jeffrey D Bradley, MD ${ }^{1}$, Michael C Roach, MD ${ }^{1,{ }^{*}}$ \\ ${ }^{1}$ Department of Radiation Oncology, Washington University School of Medicine, Saint Louis, MO \\ 63110
}

\begin{abstract}
Purpose: Optimal management of isolated local recurrences after stereotactic body radiation therapy (SBRT) for early non-small cell lung cancer (NSCLC) is unknown and literature describing repeat SBRT for in-field recurrences after initial SBRT are sparse. We investigate the safety and efficacy of salvage SBRT for isolated local failures after initial SBRT for NSCLC.

Methods/Materials: Patients receiving SBRT for isolated local recurrence after initial SBRT for early NSCLC were identified using a prospective registry. Both courses were 3-5 fractions with a biologically effective dose (BED10) of $\geq 100 \mathrm{~Gy}$. Local failure was defined as within $1 \mathrm{~cm}$ of the initial planning target volume (PTV) or an overlap of the $25 \%$ isodose lines of the first and second treatments. Failures $>1 \mathrm{~cm}$ beyond the PTV and without $\geq 25 \%$ overlap, or with additional recurrence sites were excluded. Kaplan-Meier analysis was used to estimate survival.
\end{abstract}

Results: A total 21 patients receiving salvage SBRT from 2008 to 2017 were identified. Median interval from initial SBRT to salvage SBRT was 23 months (7-52). Six patients (29\%) had central tumors. Median follow-up time from salvage SBRT was 24 months (3-60). Median overall survival after salvage was 39 months. After reirradiation, two-year primary tumor control was $81 \%$, regional nodal control was $89 \%$, distant control was $75 \%$ and overall survival was $68 \%$. Grade 2 pneumonitis occurred in 2 patients $(10 \%)$ and grade 2 chest wall toxicity in 4 patients (19\%). No grade $3+$ toxicity was observed.

Conclusions: Salvage SBRT for isolated local failures after initial SBRT appears safe, with low treatment-related toxicity and encouraging rates of tumor control.

\section{Keywords}

lung cancer; stereotactic body radiation therapy; local failure; reirradiation; salvage

*Corresponding author: Michael C Roach, M.D., Department of Radiation Oncology, Center for Advanced Medicine, Washington University School of Medicine; 4921 Parkview Place, Lower Level, St. Louis, MO 63110; Phone: (314) 362-8528; Fax: (314) 362-8521; roachm@wustl.edu.

Publisher's Disclaimer: This is a PDF file of an unedited manuscript that has been accepted for publication. As a service to our customers we are providing this early version of the manuscript. The manuscript will undergo copyediting, typesetting, and review of the resulting proof before it is published in its final form. Please note that during the production process errors may be discovered which could affect the content, and all legal disclaimers that apply to the journal pertain.

Conflict of Interest: We have no conflict of interest to disclose for this work. 


\section{Introduction}

Stereotactic body radiotherapy (SBRT) is an established treatment option in the management of patients with early-stage non-small cell lung cancer (NSCLC). Prospective studies have demonstrated that SBRT achieves high rates of local tumor control (1-3). Compared to conventionally-fractionated radiotherapy (CFRT) for early stage NSCLC, SBRT is associated with equivalent tumor control, shortened treatment times, and decreased toxicity, with larger retrospective studies even suggesting a survival benefit (4-7). Although development of distant metastases is the predominant pattern of failure after treatment with SBRT, primary tumor and local recurrences are still observed in approximately $10-15 \%$ of patients (8-10). However, salvage options for isolated post-radiation local failures are limited, with surgery or retreatment with radiotherapy as potential modalities.

While salvage surgical resection after previous thoracic irradiation has shown encouraging results, most patients experiencing post-radiation local relapse are not operative candidates (11-13). Therefore, reirradiation may be the only viable salvage option for many patients. Retreatment with CFRT has been reported for locoregional failures, with generally-poor outcomes $(14,15)$. Although salvage SBRT after initial CFRT has been described in select small series of heterogeneous patient groups ranging from early stage to locally-advanced and metastatic, few data exist to guide us on the role of reirradiation with SBRT for isolated recurrences after initial SBRT for early-stage NSCLC (16-19). Importantly, questions regarding the safety and efficacy in this setting are still largely unanswered as the few studies attempting to answer this question often include treatment of new lesions outside the initial high-dose field, which confounds the rates of both toxicity and control. The purpose of our study is to determine the tumor control and tolerability of repeat SBRT as salvage for isolated local recurrences within the high-dose volume of initial SBRT for early-stage NSCLC.

\section{Materials and Methods}

An institutional review board-approved, prospectively-maintained database was used to review patients with early-stage NSCLC initially treated with definitive SBRT who experienced isolated local recurrences salvaged with repeat SBRT. All patients opted in for inclusion. Both SBRT courses for all patients were 3-5 fractions with a biologically effective dose (BED10) of $\geq 100 \mathrm{~Gy}$. We included only patients with isolated recurrences within the high-dose region of their initial SBRT treatment, including the Radiation Therapy Oncology Group (RTOG) 0236 definition of primary tumor failure encompassing lesions within $1 \mathrm{~cm}$ of the original planning target volume (PTV), and/or new primaries with SBRT treatment having overlap of at least the $25 \%$ prescription isodose line with the initial SBRT treatment. Patients with involved lobar failures that were further than $1 \mathrm{~cm}$ from the original PTV and did not have $225 \%$ isodose line overlap were excluded from the current study. This was done due to concerns of misrepresenting both tumor control and toxicity outcomes if patients with less overlap of the two SBRT treatments were included given that lobar failures may often be far from the original lesion despite being in the same anatomic lobe. 
Patients were evaluated by a multidisciplinary team, with pretreatment evaluation including history and physical examination as well as pulmonary function testing in most patients. Medical inoperability was determined by a cardiothoracic surgeon and/or pulmonologist. At both time of initial diagnosis and salvage, patients were staged clinically with computerized tomography (CT) of the chest, positron emission tomography (PET), and/or mediastinal staging with endobronchial ultrasound (EBUS) or mediastinoscopy as indicated. Candidacy for reirradiation with repeat definitive SBRT was determined by the treating radiation oncologist after assessment of medical comorbidities, tumor location and prior organ at-risk (OAR) doses, as well as individual patient goals of care.

We have described our institutional SBRT technique in a previous manuscript (20). Abdominal compression was utilized to minimize the range of tumor motion. Daily cone beam CT was used to confirm patient setup and target verification. Delivery types included both coplanar and noncoplanar 3-dimensional conformal (3DCRT), intensity-modulated radiation therapy (IMRT), and volumetric modulated arc therapy (VMAT). For 3DCRT, prescription was typically to the $75 \%$ to $85 \%$ isodose line so that $95 \%$ of the prescribed dose covers the PTV. Plan optimization for IMRT/VMAT treatments was set to recapitulate coverage and hotspots similar to the 3DCRT plans. No specific universal dosimetric constraints were used for reirradiation, we took particular care to ensure cumulative doses to high-risk OARs, such as the spinal cord 2 Gy equivalent dose (EQD2) not exceeding $50 \mathrm{~Gy}$. No specific chest wall constraints were used in initial or salvage SBRT planning with the exception of attempting to avoid hotspots within the chest wall. Cumulative EQD2 doses to high-risk OARs were calculated by voxel-based dose accumulation.

Patients were followed with serial CT scans at 6 weeks' post-treatment, then every 3 months for 2 years, followed by every 6 months for 3 additional years. Follow up was calculated from time of salvage SBRT treatment until death or last follow-up. Primary tumor failure was defined as radiographic evidence of local enlargement of the treated primary tumor. Hilar and mediastinal nodal recurrences were considered regional failures. New lung cancers developing 5 years or greater from salvage were considered new primaries. Metachronous primary lung cancers were defined if they fulfilled one of the following criteria per the 2013 American College of Chest Physicians (ACCP) guidelines: tumor of a different histology, a tumor of the same histology but in a different lobe than the initial primary and no evidence of nodal involvement or systemic metastases, or a tumor of the same histology but temporarily separated with at least a 4-year interval between cancers and no evidence of systemic metastases (21). Toxicity was graded per the Common Terminology Criteria for Adverse Events (CTCAE), version 4.0. The Kaplan-Meier method was used to estimate local control and survival probabilities. Significance was considered at $\mathrm{p}<0.05$ and all significance levels were 2-sided. IBM® SPSS ${ }^{\circledR}$ Statistics, version 23 was used for all statistical analyses.

\section{Results}

Out of 1,209 patients in our institutional lung SBRT database, 844 patients had early stage NSCLC. Of these, 803 received an initial SBRT course with BED $>100 \mathrm{~Gy}$. Isolated local recurrence occurred in 44 of 803 patients, with 25 patients receiving SBRT reirradiation. 
Four patients receiving SBRT with BED $<100$ Gy were excluded. Therefore, we identified 21 patients receiving salvage SBRT at our institution between 2008 and 2017 who met the aforementioned inclusion and exclusion criteria. Patient, tumor, and treatment-related characteristics are summarized in Table 1. The remaining 19 patients with isolated local recurrences who did not receive repeat SBRT either died of intercurrent disease prior to retreatment (6 patients), were reirradiated with conventionally-fractionated RT due to periesophageal location (6 patients), received systemic therapy alone (5 patients), or declined reirradiation (2 patients). Compared to the 21 patients receiving salvage SBRT with BED > $100 \mathrm{~Gy}$, the 23 remaining patients with local failures had statistically similar baseline characteristics except for a larger tumor diameter at recurrence (Supplementary Table 1).Median interval between initial definitive SBRT for early-stage NSCLC and salvage SBRT for isolated local failure was 23 months ( $7-53$ months). Median age at salvage was 75 (59 - 89). No patients received out-of-field RT or previous chemotherapy prior to salvage. Reirradiation prescription dose was $50 \mathrm{~Gy}$ in 5 fractions (biological effective dose, $\left.\mathrm{BED}_{10}=100 \mathrm{~Gy}\right)$ for 12 patients and $54 \mathrm{~Gy}$ in 3 fractions $\left(\mathrm{BED}_{10}=151 \mathrm{~Gy}\right)$ for 9 patients. Figure 1 shows a representative salvage SBRT plan. There were 6 patients with central tumors per RTOG 0813 criteria, with the remaining 15 patients having peripheral tumors. All 6 central tumors were within $2 \mathrm{~cm}$ of but not directly abutting or invading the main bronchi or other mediastinal structures. No patients had tumors adjacent to the proximal bronchial tree. After salvage SBRT, no patients received adjuvant chemotherapy. Pretreatment pulmonary function tests were obtained in 14 patients (66\%). The median FEV1 was $1.5 \mathrm{~L}$ and $53 \%$ (range, $0.6-2.9 \mathrm{~L}$ and $29-109 \%$, respectively), and median DLCO was $44 \%$ (range, $35-59 \%$ ).

Cumulative dosimetric data was also obtained. Equivalent dose in 2-Gy fractions (EQD2) was calculated via voxel-based dose accumulation based on the point maximum doses $\left(D_{\max }\right)$ to adjust for variation in dose-fractionation schemes. An $\alpha / \beta$ ratio of 10 was used for tumor calculations, 3 for non-neural tissue calculations, and 2 for spinal cord and brachial plexus calculations. The median cumulative tumor EQD2/10 $\mathrm{D}_{\max }$ was $168.3 \mathrm{~Gy}(97.3-$ 266.6), median cumulative lung EQD2/3 $\mathrm{D}_{\max }$ was $286.1 \mathrm{~Gy}(137.0$ - 469.6), median cumulative cord EQD2/2 $\mathrm{D}_{\max }$ was $12.2 \mathrm{~Gy}(3.0$ - 21.1), median cumulative esophagus EQD2/3 $\mathrm{D}_{\max }$ was $18.7 \mathrm{~Gy}\left(4.9\right.$ - 37.2), median cumulative heart EQD2/3 $\mathrm{D}_{\max }$ was 21.8 Gy $\left(0.5\right.$ - 109.6), median cumulative brachial plexus EQD2/2 $\mathrm{D}_{\max }$ was $10.8 \mathrm{~Gy}(0.7$ 63.1), median cumulative lung - internal target volume (ITV) receiving $20 \mathrm{~Gy}$ (V20) was $12.0 \%$ (6.4 - 16.5), and median cumulative lung - ITV EQD2/3 mean dose was 5.5 Gy (3.0 $-9.0)$.

Median follow-up after retreatment with SBRT was 24 months (3-60 months). The median post-salvage overall survival was 39 months (95\% confidence interval [CI], 27 - 52 months). The estimated post-salvage 2-year primary tumor control was $81 \%$ (95\% CI, $60-100 \%$ ), involved lobar control was $81 \%$ (95\% CI, $60-100 \%$ ), regional nodal control was $89 \%$ (95\% CI, $71-100 \%$ ), distant control was $75 \%$ (95\% CI, $57-100 \%$ ), and overall survival was $68 \%$ (95\% CI, $51-96 \%$ ) for patients (Figure 2). The patterns of failure among study patients is shown in Table 2, with distant failure being the most common sites of treatment failure. There were zero isolated local recurrences after salvage SBRT. However, there were two patients who failed locally and also had either a nodal or distant failure concomitantly. 
Four patients developed biopsy-proven metachronous lung primaries after salvage at a median time of 19.5 months post-SBRT. Of these, 3 were new early-stage NSCLC, 1 in a separate lobe of the ipsilateral lung and 2 in the contralateral lung, while 1 was limited-stage small cell lung cancer in the contralateral lung.

Treatment-related adverse events were relatively low, with zero grade $3+$ toxicity observed in this series. Two patients developed symptomatic grade 2 radiation pneumonitis, managed with steroids. Grade 3+ pneumonitis was not observed in this cohort. Chest wall toxicity developed in four patients, two of which were rib fractures, which was all managed conservatively with over-the-counter medication. Non-pneumonitis pulmonary toxicity, esophagitis or significant skin toxicity was not observed. Only 3 patients had post-treatment pulmonary function testing, however, with minimal changes observed in these patients compared to baseline.

\section{Discussion}

Herein, we report our institutional experience of salvage SBRT for isolated local relapse after initial SBRT for early stage NSCLC. We achieved promising local and distant control rates after salvage, as well as favorable survival in this medically-inoperable population with significant comorbidities. Importantly, we observed acceptably low rates of pneumonitis and chest wall toxicity at extended follow-up. We observed no esophagitis or bronchial tree toxicity in centrally-located tumors. However, no patients in our series had "ultra-central" tumors, defined as directly abutting or invading the proximal bronchial tree (22). This is particularly encouraging given the highly-selected cohort of patients with local recurrence within the high-dose volume of an initial SBRT course with a $\mathrm{BED}_{10}$ of at least $100 \mathrm{~Gy}$.

Despite high rates of local control in patients receiving of commonly-employed regimens of $\mathrm{BED}_{10}$ of $100 \mathrm{~Gy}$ or greater, local recurrence after definitive SBRT is not uncommon, with actuarial rates of up to $20 \%$ in large series with long-term follow-up $(23,24)$. Therefore, delineating the optimal salvage options for these patients is of utmost importance. However, options for salvage in patients experiencing local failures after initial SBRT are both limited and poorly-characterized. Several reports have published high rates of tumor control with acceptably low toxicity after salvage lobectomy for local recurrences after being treated with upfront SBRT, with isolated reports of fistula formation being the primary life-threatening adverse event and 3-year survival greater than 70\% (11-13, 25). Even with these encouraging results, salvage surgical resection is a safe option in only a select subset of favorable patients initially treated for SBRT. Even in initially operable patients treated with upfront SBRT, only approximately $20 \%$ of patients with a local recurrence undergo salvage lung resection $(11,25)$. Therefore, for the majority of patients, radiotherapy remains the only definitive salvage option in this setting.

Conventionally-fractionated reirradiation with either photon or proton therapy has been previously reported after initial conventional RT, for recurrent locally-advanced or oligometastatic NSCLC, with acceptable local control rates but relatively high rates of pneumonitis $(14,26-28)$. Interstitial brachytherapy with iodine-125 seeds for intrathoracic recurrences after conventional RT have also been reported, although in a small group of 
heterogeneous patients with both primary lung tumors and pulmonary metastases (29). A more recent series describing intensity-modulated proton therapy (IMPT) for thoracic reirradiation to a median 66 cobalt-Gy equivalent in 33 fractions demonstrate durable local control with no grade 4 or 5 toxicity and low levels of pneumonitis compared to previous reports, suggesting this may be a promising option for locally advanced intrathoracic recurrences at centers with access to IMPT (30).

SBRT for thoracic reirradiation has been previously described, almost exclusively in the setting of previous conventionally-fractionated RT for a heterogeneous group of early stage to metastatic lung cancer, with a wide range of local control and survival as well as toxicity likely owing to a wide range of initial and retreatment doses, broad patient inclusion, and systemic therapy use (16,31-33). However, our understanding of the role of SBRT after initial SBRT is even less well-defined. The few studies evaluating this frequently include patients with metastatic disease, often use regimens greater than 5 fractions with $\mathrm{BED}_{10}$ less than $100 \mathrm{~Gy}$, are intermixed with conventionally-fractionated regimens, and are usually in the situation of treating out-of-field intrathoracic failures (34-37). Even in more carefullycontrolled studies including marginal or intralobar failures, new same-lobe lesions may be far away from the initial high-dose volume and can therefore underrepresent toxicity risk and overestimate control rates (19). Still, these provide important preliminary work in understanding feasibility and outcomes for repeat SBRT in a wide array of therapeutic settings. To our knowledge, the series by Hearn and colleagues and two cases reported by Nishimura et al are the only previous studies reporting outcomes of SBRT for salvage of isolated local recurrence after initial SBRT with both courses' $\mathrm{BED}_{10} \geq 100 \mathrm{~Gy}$ for early stage NSCLC $(18,38)$. In 10 patients with a median follow-up of 13.8 months, 2 patients developed distant failure and 4 had local failure, with no grade 3-5 toxicity observed. Our results in 21 patients are comparable to Hearn et al in terms of control and safety, although distant metastases were still the predominant pattern of failure in our series with extended follow-up, akin to patterns in the upfront SBRT setting for early $\operatorname{NSCLC}(8,9)$.

One of the primary concerns of thoracic reirradiation has been of potentially life-threatening treatment-related toxicity, particularly in centrally-located tumors. Fatal bleeding in central tumors and a case of gastric perforation in peripheral lower lobe tumors have previously been reported $(34,39)$. We report zero grade $3+$ toxicity, including zero bronchial tree toxicity or esophagitis in the 6 patients with centrally-located tumors. High rates of symptomatic pneumonitis have been reported after thoracic reirradiation, particularly with the use of conventionally-fractionated radiation therapy, with the volume receiving 5 Gy (V5) and 20 Gy (V20), both predicting for pneumonitis (31, 40). Accordingly, out-of-field failures are associated with higher rates of pneumonitis than in-field recurrences, which may well be due to the increased low-dose volume of lung parenchyma and may at least partially explain why we observed relatively low rates of pneumonitis. Chest wall toxicity was the most common adverse event in our study. In the upfront SBRT setting, the volume of irradiated chest wall, maximum dose to the chest wall, and prescription dose and fractionation all predict for chest wall toxicity, although no similar predictors currently exist after reirradiation (41-43). While we aim to avoid hotspots in the chest wall during the planning process, specific chest wall constraints are not routinely used in our treatment planning process, which may influence the rates observed in the current series. 
Although the strengths of our study include the relatively large number of patients and strict inclusion criteria, there are considerable limitations. At least $25 \%$ isodose overlap was required to minimize confounding of toxicity and efficacy by lesions without high-dose overlap at time of reirradiation. However, there can still be considerable distance between the two PTVs while maintaining at least $25 \%$ isodose overlap, although this distance may be much less than if a same lobe or same lung criterion was used. Furthermore, our encouraging outcomes may be influenced by selection bias, since not all local failures after initial SBRT received reirradiation. Given the retrospective design, grade 1-2 toxicity may be under-reported, further influenced by the fact that adverse events were physicianreported, as patient-reported quality of life outcomes could more accurately assess treatment effects. Although pre-treatment PFTs were obtained on most patients, only 3 patients had post-SBRT testing performed, so the true effect on pulmonary function after repeat SBRT is unknown. Although our follow-up of 24 months is longer than most comparable reirradiation series in this high-risk cohort, late adverse events may be inadequately captured. Accordingly, estimates of tumor control may be significantly affected with a longer study duration. Furthermore, we have evolved our institutional SBRT techniques over the study period, and although most study patients were treated with noncoplanar 3DCRT, VMAT is being increasingly utilized in our clinic for lung SBRT. Despite these limitations, reirradiation with SBRT appears to be a safe and effective salvage option for isolated local failures after initial SBRT.

For patients with early-stage NSCLC treated with definitive SBRT experiencing isolated local failure, reirradiation with definitive-dose SBRT appears to be feasible and welltolerated. For medically-inoperable patients with local relapse in early-stage NSCLC, repeat SBRT should be considered as a potential salvage option and warrants further investigation, although caution should be taken, particularly with centrally-located tumors.

\section{Supplementary Material}

Refer to Web version on PubMed Central for supplementary material.

\section{Funding:}

Supported by Clinical and Translational Science Award (CTSA) Grant [UL1 TR000448] and Siteman Comprehensive Cancer Center and NCI Cancer Center Support Grant P30 CA091842

\section{References}

1. Chang JY, Senan S, Paul MA, et al. Stereotactic ablative radiotherapy versus lobectomy for operable stage I non-small-cell lung cancer: a pooled analysis of two randomised trials. Lancet Oncol. 2015;16:630-637. [PubMed: 25981812]

2. Timmerman RD, Paulus R, Pass HI, et al. Stereotactic Body Radiation Therapy for Operable EarlyStage Lung Cancer: Findings From the NRG Oncology RTOG 0618 Trial. JAMA Oncol. 2018;4:1263-1266. [PubMed: 29852037]

3. Timmerman RD, Hu C, Michalski JM, et al. Long-term Results of Stereotactic Body Radiation Therapy in Medically Inoperable Stage I Non-Small Cell Lung Cancer. JAMA Oncol. 2018;4:1287-1288. [PubMed: 29852036] 
4. Jeppesen SS, Schytte T, Jensen HR, et al. Stereotactic body radiation therapy versus conventional radiation therapy in patients with early stage non-small cell lung cancer: an updated retrospective study on local failure and survival rates. Acta Oncol. Stockh. Swed 2013;52:1552-1558.

5. Nyman J, Hallqvist A, Lund J-Å, et al. SPACE - A randomized study of SBRT vs conventional fractionated radiotherapy in medically inoperable stage I NSCLC. Radiother. Oncol. J. Eur. Soc. Ther. Radiol. Oncol 2016;121:1-8.

6. Haque W, Verma V, Polamraju P, et al. Stereotactic body radiation therapy versus conventionally fractionated radiation therapy for early stage non-small cell lung cancer. Radiother. Oncol. J. Eur. Soc. Ther. Radiol. Oncol 2018.

7. Reibnitz D von, Shaikh F, Wu AJ, et al. Stereotactic body radiation therapy (SBRT) improves local control and overall survival compared to conventionally fractionated radiation for stage I non-small cell lung cancer (NSCLC). Acta Oncol. 2018;0:1-7.

8. Bradley JD, El Naqa I, Drzymala RE, et al. Stereotactic Body Radiation Therapy for Early-Stage Non-Small-Cell Lung Cancer: The Pattern of Failure Is Distant. Int. J. Radiat. Oncol 2010;77:1146-1150.

9. Andratschke N, Zimmermann F, Boehm E, et al. Stereotactic radiotherapy of histologically proven inoperable stage I non-small cell lung cancer: Patterns of failure. Radiother. Oncol 2011;101:245249. [PubMed: 21724287]

10. Stephans KL, Woody NM, Reddy CA, et al. Tumor Control and Toxicity for Common Stereotactic Body Radiation Therapy Dose-Fractionation Regimens in Stage I Non-Small Cell Lung Cancer. Int. J. Radiat. Oncol 2018;100:462-469.

11. Hamaji M, Chen F, Matsuo Y, et al. Treatment and Prognosis of Isolated Local Relapse after Stereotactic Body Radiotherapy for Clinical Stage I Non-Small-Cell Lung Cancer: Importance of Salvage Surgery. J. Thorac. Oncol. Off. Publ. Int. Assoc. Study Lung Cancer 2015;10:1616-1624.

12. Verstegen NE, Maat APWM, Lagerwaard FJ, et al. Salvage surgery for local failures after stereotactic ablative radiotherapy for early stage non-small cell lung cancer. Radiat. Oncol. Lond. Engl 2016;11:131.

13. Antonoff MB, Correa AM, Sepesi B, et al. Salvage pulmonary resection after stereotactic body radiotherapy: A feasible and safe option for local failure in selected patients. J. Thorac. Cardiovasc. Surg 2017;154:689-699. [PubMed: 28495066]

14. Wu K-L, Jiang G-L, Qian H, et al. Three-dimensional conformal radiotherapy for locoregionally recurrent lung carcinoma after external beam irradiation: A prospective phase I-II clinical trial. Int. J. Radiat. Oncol 2003;57:1345-1350.

15. Tada T, Fukuda H, Matsui K, et al. Non-small-cell lung cancer: reirradiation for locoregional relapse previously treated with radiation therapy. Int. J. Clin. Oncol 2005;10:247-250. [PubMed: 16136369]

16. Kelly P, Balter PA, Rebueno N, et al. Stereotactic body radiation therapy for patients with lung cancer previously treated with thoracic radiation. Int. J. Radiat. Oncol. Biol. Phys 2010;78:13871393. [PubMed: 20381271]

17. Kilburn JM, Kuremsky JG, Blackstock AW, et al. Thoracic re-irradiation using stereotactic body radiotherapy (SBRT) techniques as first or second course of treatment. Radiother. Oncol 2014;110:505-510. [PubMed: 24444530]

18. Hearn JWD, Videtic GMM, Djemil T, et al. Salvage Stereotactic Body Radiation Therapy (SBRT) for Local Failure After Primary Lung SBRT. Int. J. Radiat. Oncol 2014;90:402-406.

19. Horne ZD, Dohopolski MJ, Clump DA, et al. Thoracic reirradiation with SBRT for residual/ recurrent and new primary NSCLC within or immediately adjacent to a prior high-dose radiation field. Pract. Radiat. Oncol 2018;8:e117-e123. [PubMed: 29724402]

20. Nikitas J, DeWees T, Rehman S, et al. Stereotactic Body Radiotherapy for Early-Stage Multiple Primary Lung Cancers. Clin. Lung Cancer 2018.

21. Kozower BD, Larner JM, Detterbeck FC, et al. Special Treatment Issues in Non-small Cell Lung Cancer: Diagnosis and Management of Lung Cancer, 3rd ed: American College of Chest Physicians Evidence-Based Clinical Practice Guidelines. CHEST. 2013;143:e369S-e399S. [PubMed: 23649447] 
22. Chaudhuri AA, Tang C, Binkley MS, et al. Stereotactic ablative radiotherapy (SABR) for treatment of central and ultra-central lung tumors. Lung Cancer. 2015;89:50-56. [PubMed: 25997421]

23. Onishi H, Shirato H, Nagata Y, et al. Hypofractionated stereotactic radiotherapy (HypoFXSRT) for stage I non-small cell lung cancer: updated results of 257 patients in a Japanese multi-institutional study. J. Thorac. Oncol. Off. Publ. Int. Assoc. Study Lung Cancer 2007;2:S94-100.

24. Shintani T, Matsuo Y, Iizuka Y, et al. A Retrospective Long-term Follow-up Study of Stereotactic Body Radiation Therapy for Non-Small Cell Lung Cancer From a Single Institution: Incidence of Late Local Recurrence. Int. J. Radiat. Oncol 2018;100:1228-1236.

25. Chen F, Matsuo Y, Yoshizawa A, et al. Salvage Lung Resection for Non-small Cell Lung Cancer After Stereotactic Body Radiotherapy in Initially Operable Patients. J. Thorac. Oncol 2010;5:1999-2002. [PubMed: 21102261]

26. Kruser TJ, McCabe BP, Mehta MP, et al. Reirradiation for Locoregionally Recurrent Lung Cancer Outcomes in Small Cell and Non-Small Cell Lung Carcinoma. Am. J. Clin. Oncol 2014;37:70-76. [PubMed: 23357968]

27. McAvoy S, Ciura K, Wei C, et al. Definitive Reirradiation for Locoregionally Recurrent Non-Small Cell Lung Cancer With Proton Beam Therapy or Intensity Modulated Radiation Therapy: Predictors of High-Grade Toxicity and Survival Outcomes. Int. J. Radiat. Oncol 2014;90:819-827.

28. Chao H-H, Berman AT, Simone CB, et al. Multi-Institutional Prospective Study of Reirradiation with Proton Beam Radiotherapy for Locoregionally Recurrent Non-Small Cell Lung Cancer. J. Thorac. Oncol 2017;12:281-292. [PubMed: 27826034]

29. Jiang P, Liu C, Wang J, et al. Computed tomography (CT)-guided interstitial permanent implantation of (125)I seeds for refractory chest wall metastasis or recurrence. Technol. Cancer Res. Treat 2015;14:11-18. [PubMed: 24502546]

30. Ho JC, Nguyen Q-N, Li H, et al. Reirradiation of thoracic cancers with intensity modulated proton therapy. Pract. Radiat. Oncol 2018;8:58-65. [PubMed: 28867546]

31. Liu H, Zhang X, Vinogradskiy YY, et al. Predicting Radiation Pneumonitis After Stereotactic Ablative Radiation Therapy in Patients Previously Treated With Conventional Thoracic Radiation Therapy. Int. J. Radiat. Oncol. Biol. Phys 2012;84:1017-1023. [PubMed: 22543216]

32. Reyngold M, Wu AJ, McLane A, et al. Toxicity and outcomes of thoracic re-irradiation using stereotactic body radiation therapy (SBRT). Radiat. Oncol 2013;8:99. [PubMed: 23617949]

33. Trovo M, Minatel E, Durofil E, et al. Stereotactic Body Radiation Therapy for Re-irradiation of Persistent or Recurrent Non-Small Cell Lung Cancer. Int. J. Radiat. Oncol 2014;88:1114-1119.

34. Peulen H, Karlsson K, Lindberg K, et al. Toxicity after reirradiation of pulmonary tumours with stereotactic body radiotherapy. Radiother. Oncol 2011;101:260-266. [PubMed: 22056534]

35. Valakh V, Miyamoto C, Micaily B, et al. Repeat stereotactic body radiation therapy for patients with pulmonary malignancies who had previously received SBRT to the same or an adjacent tumor site. J. Cancer Res. Ther 2013;9:680-685. [PubMed: 24518717]

36. Meijneke TR, Petit SF, Wentzler D, et al. Reirradiation and stereotactic radiotherapy for tumors in the lung: Dose summation and toxicity. Radiother. Oncol 2013;107:423-427. [PubMed: 23647748]

37. Ogawa Y, Shibamoto Y, Hashizume C, et al. Repeat stereotactic body radiotherapy (SBRT) for local recurrence of non-small cell lung cancer and lung metastasis after first SBRT. Radiat. Oncol. Lond. Engl 2018;13.

38. Nishimura S, Takeda A, Sanuki N, et al. Dose-Escalated Stereotactic Body Radiotherapy (SBRT) as a Salvage Treatment for Two Cases with Relapsed Peripheral Lung Cancer After Initial SBRT. J. Thorac. Oncol 2015;10:e69-e71. [PubMed: 26200281]

39. Nonaka H, Onishi H, Ozaki M, et al. Serious gastric perforation after second stereotactic body radiotherapy for peripheral lung cancer that recurred after initial stereotactic body radiotherapy: a case report. J. Med. Case Reports 2017;11:343.

40. Ren C, Ji T, Liu T, et al. The risk and predictors for severe radiation pneumonitis in lung cancer patients treated with thoracic reirradiation. Radiat. Oncol. Lond. Engl 2018;13.

41. Dunlap NE, Cai J, Biedermann GB, et al. Chest Wall Volume Receiving > 30 Gy Predicts Risk of Severe Pain and/or Rib Fracture After Lung Stereotactic Body Radiotherapy. Int. J. Radiat. Oncol 2010;76:796-801. 
42. Mutter RW, Liu F, Abreu A, et al. Dose-Volume Parameters Predict for the Development of Chest Wall Pain After Stereotactic Body Radiation for Lung Cancer. Int. J. Radiat. Oncol. Biol. Phys 2012;82:1783-1790. [PubMed: 21868173]

43. Ma J-T, Liu Y, Sun L, et al. Chest wall toxicity after stereotactic body radiation therapy: a pooledanalysis of 57 studies. Int. J. Radiat. Oncol 2018. 


\section{Highlights:}

- Local recurrences occur in $10-15 \%$ of early stage NSCLC after definitive SBRT

- $\quad$ Salvage SBRT safety and efficacy for local recurrence after initial SBRT is unknown

- $\quad$ Median overall survival after salvage SBRT was 39 months in 21 patients

- Primary tumor and distant control at 2 years were $81 \%$ and $75 \%$ after salvage

- We observed no grade 3+ AEs, with grade 1-2 pneumonitis in $10 \%$ and CW pain in $19 \%$ 

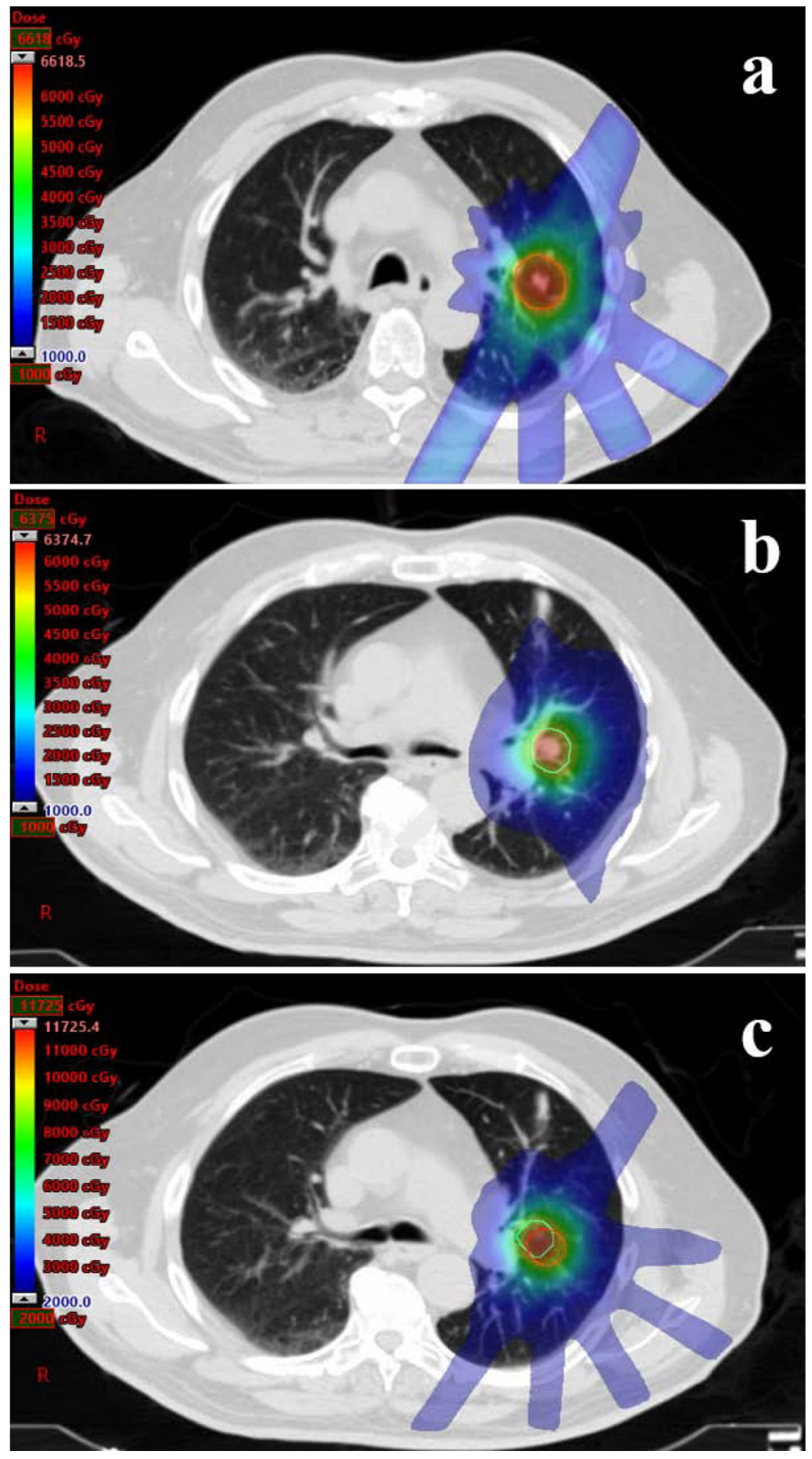

Figure 1.

Prescription isodose distributions for a patient treated with salvage SBRT to 50Gy in 5 fractions for an isolated local recurrence after initial SBRT to 54Gy in 3 fractions. The planning target volume (PTV) of the initial plan (a) is indicated by the red line. The PTV of the salvage plan (b) is indicated by the green line. The composite plan (c) with both PTVs is displayed, with a maximum cumulative point dose of $117 \mathrm{~Gy}$. 


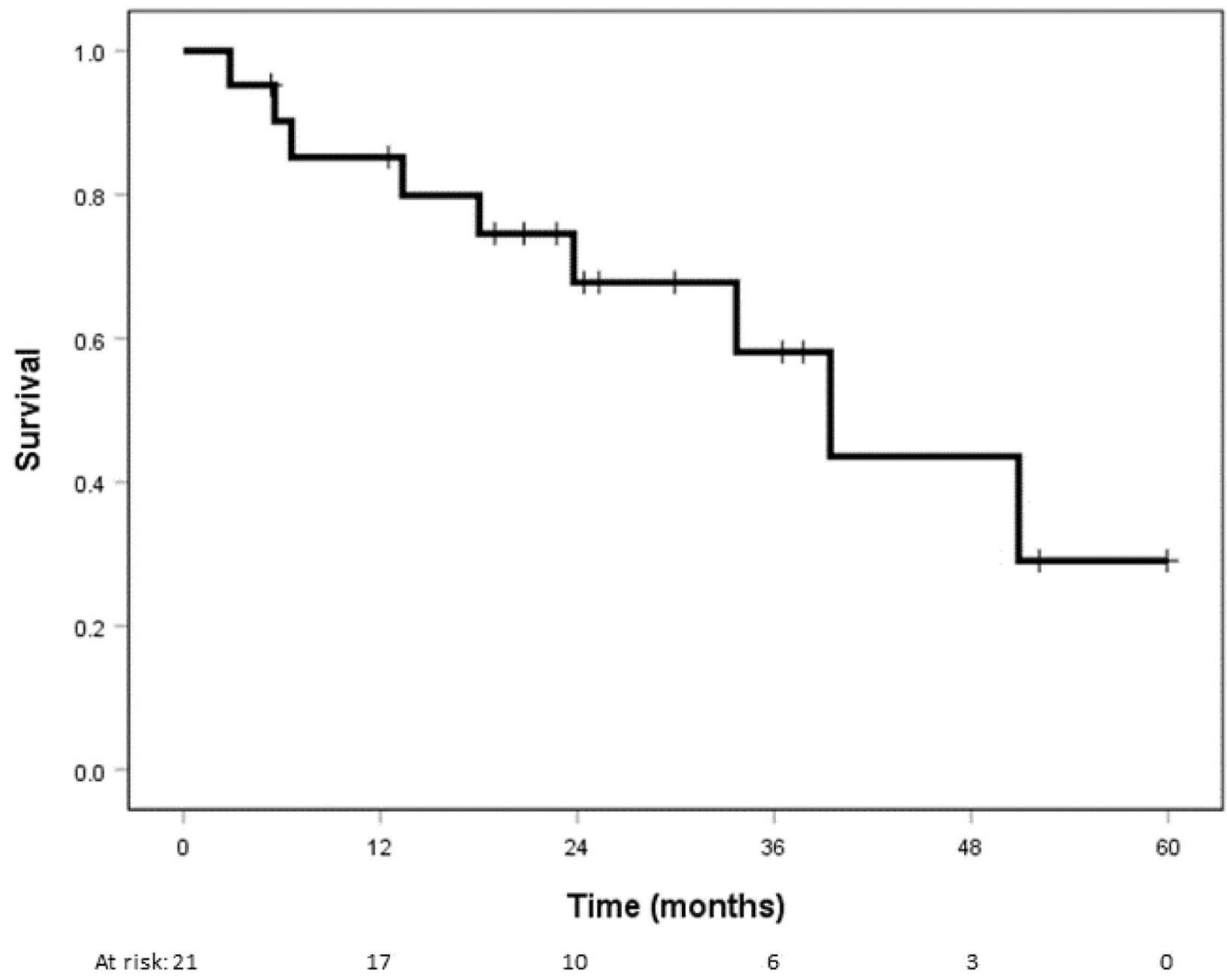

Figure 2.

Kaplan-Meier curve for overall survival. 
Table 1.

Patient- and treatment-related characteristics $(n=21)$

\begin{tabular}{|l|l|}
\hline Characteristic & Value (\% or range) \\
\hline Age at salvage (years), median & $75(59-89)$ \\
\hline Sex & \\
\hline Male & $13(62 \%)$ \\
\hline Female & $8(38 \%)$ \\
\hline BMI, median & $26(18-37)$ \\
\hline Smoking status & \\
\hline Never & $1(5 \%)$ \\
\hline Former & $9(43 \%)$ \\
\hline Current & $11(52 \%)$ \\
\hline Smoking pack-years, median & $55(27-110)$ \\
\hline Age-adjusted CCI, median & $6(2-11)$ \\
\hline KPS, median & $80(60-100)$ \\
\hline Histology & $12(57 \%)$ \\
\hline Squamous cell carcinoma & $9(43 \%)$ \\
\hline Adenocarcinoma & $7(33 \%)$ \\
\hline NSCL NOS & $5(24 \%)$ \\
\hline Biopsy unable to be obtained & $3(14 \%)$ \\
\hline Initial tumor diameter (cm), median & $6(29 \%)$ \\
\hline Tumor diameter at salvage (cm), median & $2.0(0.9-3.6)$ \\
\hline Initial PET SUV, median & $5.4(1-2.5)$ \\
\hline PET SUV at salvage, median & $6.2(2.3-22)$ \\
\hline Central location & $6(29 \%)$ \\
\hline Mediastinal staging & $19(90 \%)$ \\
\hline PET alone & \\
\hline EBUS & $15 \%)$ \\
\hline Mediastinoscopy & \\
\hline Initial dose and fractionation & \\
\hline 50 Gy in 5 fractions & \\
\hline 54 Gy in 3 fractions & \\
\hline Salvage dose and fractionation & \\
\hline 50 Gy in 5 fractions & \\
\hline 54 Gy in 3 fractions & \\
\hline
\end{tabular}

BMI, body mass index; CCI, Charlson comorbidity index; KPS, Karnofsky performance status, NSCLC NOS, non-small cell lung cancer not otherwise specific; PET, positron emission tomography; SUV, standardized uptake value; EBUS, endobronchial ultrasound 
Table 2.

Patterns of failure after salvage SBRT among study patients ( $\mathrm{n}=21)$

\begin{tabular}{|l|l|l|}
\hline Failure type & Number & Time to event, median (range) \\
\hline Local & 2 & 22.4 months $(21.3-23.5)$ \\
\hline Local only & 0 & \\
\hline Local and nodal & 1 & \\
\hline Local and distant & 1 & \\
\hline Nodal & 1 & 23.5 months \\
\hline Nodal and local & 1 & \\
\hline Distant & 4 & 12.2 months $(1.5-21.4)$ \\
\hline Distant only & 3 & \\
\hline Distant and local & 1 & \\
\hline Metachronous lung & 4 & 19.5 months $(12.0-59.3)$ \\
\hline
\end{tabular}

